\title{
Surface-anisotropic Polystyrene Spheres by Electroless Deposition
}

\author{
Jing-Qin Cui and Ilona Kretzschmar* \\ The City College of New York, Chemical Engineering Department, Steinman Hall, \\ 140th St. \& Convent Ave. New York, NY, 10031
}

Figure s1 shows a representative energy dispersive X-ray analysis spectrum (EDAX, 30 $\mathrm{mm}^{2}$ ) obtained from the AgNP-sphere/PDMS composite (Fig. s1, inset). The spectrum shows peaks for Ag (2\%), C (71\%), O (13\%), and Si (14\%). The Si and O peaks are attributed to the PDMS mask, while the carbon and silver signals arise from the sPS spheres and the silver deposit, respectively. An EDAX scan of the empty PDMS mask (Fig. s2, inset) after removal of the modified sPS spheres with a double-sided adhesive carbon tape yields Ag (<0.5\%), C (43\%), O (30\%), and Si (27\%) peaks (Fig. s2). The residual silver signal is attributed to a few silver-modified spheres that remain in the PDMS mask. 
Figure s1

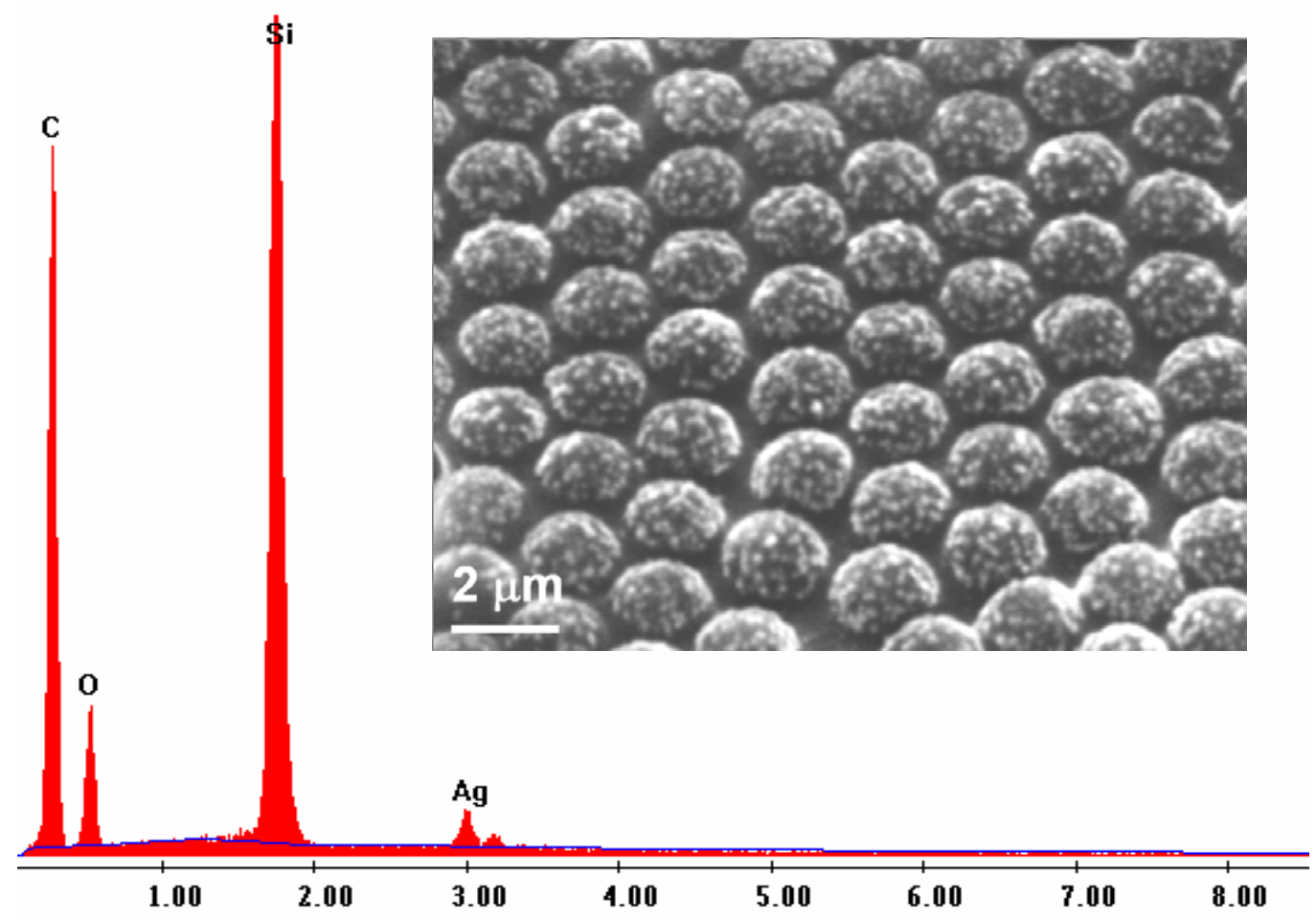

Figure s2

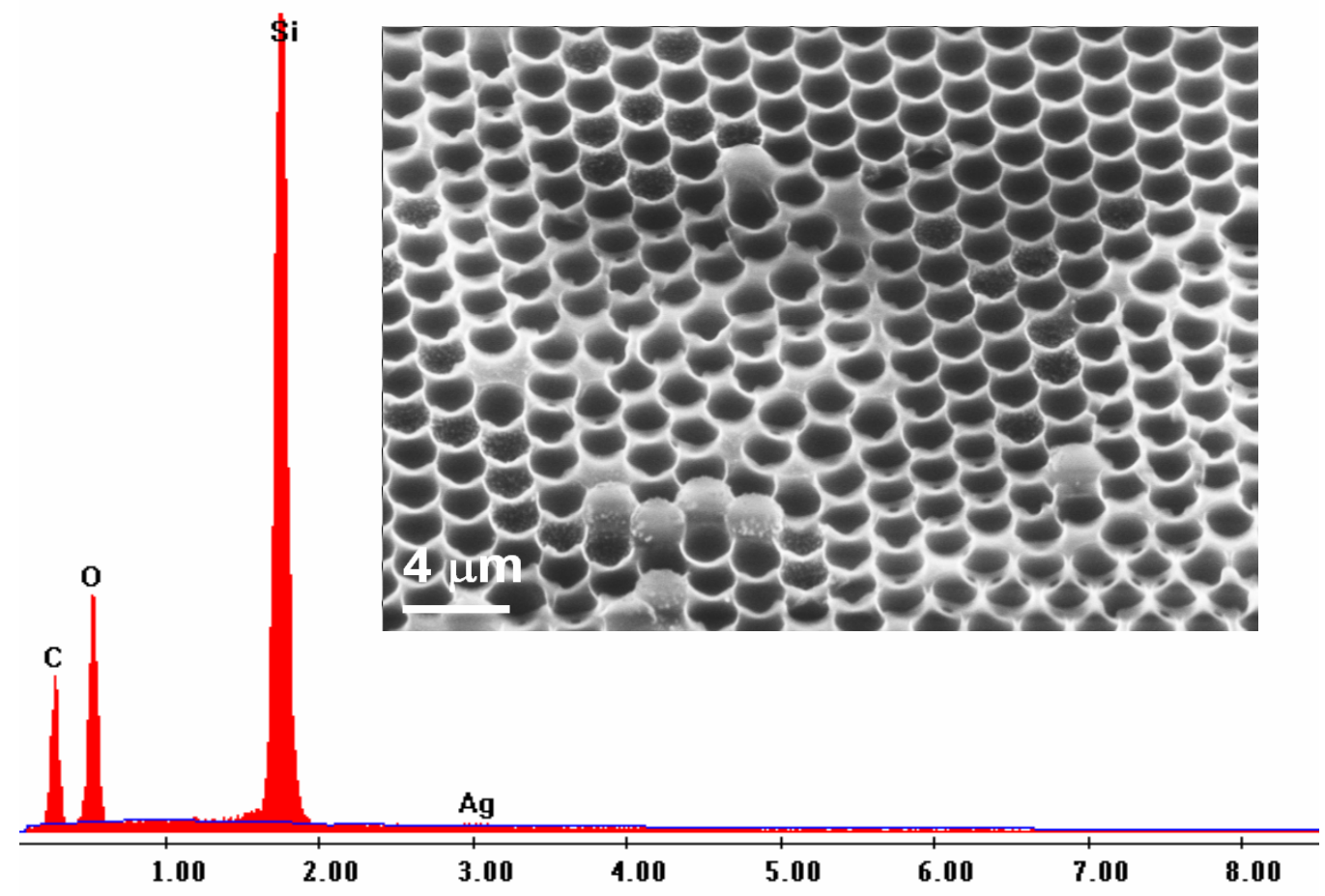

